\title{
Reproduction of Nearby Sound Sources Using a Modified Distance Coding in Higher Order Ambisonics
}

\author{
Wenbei Wang, Maoshen Jia ${ }^{*}$ Jundai Sun and Jiaming Zhang \\ Speech and Audio Signal Processing Laboratory, School of Electronic \\ Information and Control Engineering, Beijing University of Technology, Beijing \\ 100124, China, \\ wwb@emails.bjut.edu.cn, jiamaoshen@bjut.edu.cn, \\ sunjundai@emails.bjut.edu.cn, zhangjiaming@emails.bjut.edu.cn.
}

\begin{abstract}
Reproduction of nearby sound sources which include multiple frequencies is a fundamental problem in acoustic signal processing. In practical realization, the multifrequency sound source and near-field distortion are essential issues that must be considered in sound field reproduction system. As a consequence, based on the cylindrical harmonic expansion of higher order Ambisonics (HOA), a modified distance coding method for the nearby multi-frequency source reproduction is proposed in this paper. Firstly, the cylindrical harmonic expansion of multi-frequency sound source is derived in this paper. To restrict the near-field distortion for nearby sound sources, a modified distance coding is proposed. Then, by introducing near-field compensation (NFC) into the encoding stage, the modified cylindrical harmonic expansion of the nearby multi-frequency source is obtained. Finally, the match relationship between the reproduction sound field and the desired sound field is established by cylindrical harmonic analysis; besides, the weights of loudspeakers are designed by using least squares method. Simulation results show that the proposed method can accurately reproduce multi-frequency sound field; meanwhile, it can be seen that near-field distortion is evidently eliminated by the proposed method.
\end{abstract}

Keywords: Nearby sound sources, multi-frequency sound source, modified distance coding method, near-field distortion

\section{Introduction}

Sound field reproduction aims at giving one or more listeners the impression of being immersed in a realistic sound environment by the array of loudspeakers. It has been frequently used in virtual auditory environments, such as multimedia environments, auditory display, vehicle simulators, etc. According to the psychoacoustic theory [1], the human ear is sensitive to the horizontal sound field at the same height, especially for the nearby sound sources which are also known as focused sound sources [2] (typically located within $1 \mathrm{~m}$ from the listener). Hence, the reproduction technique of 2-dimensional (2D) (i.e., height invariant sound fields) nearby sound sources is more meaningful in practical situations.

So far, the implementation of nearby sound source reproduction mainly utilizes wave field synthesis (WFS) method [3], spectral division method (SMD) [4] and higher order Ambisonics (HOA) approach [5-7]. In WFS method, two separate stationary phase approximations are employed by using the Kirchhoff-Helmholtz integral equation. However, the aperture between loudspeakers is limited by the spatial sampling process; meanwhile, the required number of loudspeakers is enormous over large areas. SMD

* Corresponding Author 
formulates the nearby sound sources in wavenumber domain with employment of linear distributions of secondary sources. But this method is only employed in linear distribution of secondary sources and has a restriction on the position of sound source. Recently, HOA has been related to the implementation of spatial near-field sound reproduction, where HOA is based on the cylindrical/spherical harmonic representations of a sound field. In this method, the typical study of nearby sound sources reproduction by Daniel [6-7], in which he adopts distance coding method of nearby sound sources to restrict near-field distortion. By using this method, the sound field can be accurately reproduced in the sweet spot. However, the distance coding method brings with it a strong low-frequency boost which leads to extremely large loudspeaker signals. More recently, a series of regularization functions (RFs) such as high-pass filters [8] and cosine-shaped weighting functions [9] are employed to compensate the near-field distortion [10]. But a preliminary study shows that the performances of these RFs are found to be insufficient and the choice of the RFs impacts the reproduced sound field. Otherwise, the existing sound reproduction systems mostly reproduce monochromatic sound field; nevertheless, for real virtual auditory applications, it is necessary to consider signals which include multiple frequencies rather than single independent monochromatic sound sources.

In this paper, to address the above issues, the cylindrical harmonics expansion of the multi-frequency source is derived in this paper. Thereafter, a modified distance coding method is proposed to restrict the near-field distortion. Simulation results show that the proposed method can accurately reproduce multi-frequency sound field; besides, it can be observed that near-field distortion is evidently eliminated by the proposed method.

The remainder of this paper is organized as follows. Section 2 presents the proposed method of nearby sound sources reproduction. Section 3 provides the simulation results, while conclusions are drawn in Section 4.

\section{Proposed Method of Nearby Sound Sources Reproduction}

In practical situation, there have been various methods for sound field reproduction. Almost all of them only consider a single frequency sound source. However, reproduction of multi-frequency sources (i.e., speech signal \& audio signal) is an important issue which should be considered in acoustic signal processing. Hence, the multi-frequency sound field is firstly analyzed and the cylindrical harmonic expansion is get. In addition, in natural sound fields there are always more or less near field sources and the modeling of the near-field effect due to finite distance sources is rarely addressed in literature. Nevertheless, it points out a fundamental issue of natural or realistic sound fields. In traditional Ambisonics system, the near-field reproduction of the multi-frequency sound source results in near-field distortion. In order to eliminate the effect of high-order components [10] which mainly causes near field distortion, this section proposes a modified distance coding scheme. In Subsection 2.1, the multi-frequency desired sound field is presented by cylindrical harmonic expansion. Then, a new distance coding method is introduced to near-field synthesis in Subsection 2.2. The Subsection 2.3 calculates the weights of loudspeakers based on least-squares method and the Subsection 2.4 analyzes the influence of sound source position for near-field reproduction.

\subsection{Cylindrical Harmonic Expansion of the Multi-frequency Sound Field}

This paper mainly concentrates on the reproduction of height invariant multifrequency sound fields which are radiated by multi-frequency sound sources. At physical level, the multi-frequency sound field can be represented by acoustic pressure within a given region of space. In this subsection, the cylindrical harmonic analysis for the desired multi-frequency sound field will be discussed.

In a source free region of space, according to the acoustic linear supposition, a multifrequency sound field can be generated by multiple single-frequency sound sources. For a 
given frequency set $\boldsymbol{K}=\left\{k_{1}, k_{2}, \ldots, k_{S}\right\}$, the acoustic pressure of the multi-frequency sound field can be obtained as follow:

$$
P_{d}(\boldsymbol{x})_{K}=\sum_{s=1}^{S} \lambda_{s} P_{s}\left(\boldsymbol{x}, k_{s}\right)
$$

where $P_{s}\left(\boldsymbol{x}, k_{s}\right)$ denote the acoustic pressure of the $s^{\text {th }}$ single-frequency sound field. $\boldsymbol{x}=(r, \phi)$ is the observation point. In equation (1), the desired sound field consists of $S$ different single-frequency sound sources. $k_{s}$ is the wavenumber satisfied $k_{s}=2 \pi f_{s} / c$, where $f_{s}$ is the frequency of the $s^{\text {th }}$ sound source and $c$ is the speed of sound. $\lambda_{s}$ is the amplitude of the $s^{\text {th }}$ sound source.

All the single-frequency sound sources located at $\boldsymbol{x}_{s}=\left(r_{s}, \phi_{s}\right)$ are monopole line sources [11]. So the formula (1) can be rewritten as:

$$
P_{d}(\boldsymbol{x})_{K}=\sum_{s=1}^{S} \lambda_{s} \frac{i}{4} H_{0}^{(2)}\left(k_{s}\left|\boldsymbol{x}-\boldsymbol{x}_{s}\right|\right)
$$

By using the addition theorem of cylindrical harmonics [11-12], the multi-frequency sound field can be rewritten as:

$$
P_{d}(\boldsymbol{x})_{K}=\sum_{s=1}^{S} \sum_{m=-\infty}^{\infty} \alpha_{m}^{s}\left(k_{s}\right) J_{m}\left(k_{s} r\right) e^{i m \phi}
$$

where $H_{m}(\bullet)$ and $J_{m}(\bullet)$ represent the $m^{\text {th }}$ order Hankel function of the second kind and the $m^{\text {th }}$ order Bessel function of the first kind, respectively. Ambisonic directional encoding format assumes that virtual sound sources are in far field. Therefore, based on the cylindrical harmonic decomposition of $s^{\text {th }}$ line source of incidence $\phi_{s}$ conveying a signal $\psi_{s}$, the encoding coefficients can be represented as:

$$
\alpha_{m}^{s}\left(k_{s}\right)=\psi_{s} E_{m}\left(\phi_{s}\right)
$$

where $E_{m}\left(\phi_{s}\right)=e^{-i m \phi_{s}}, \psi_{s}=\lambda_{s} \frac{i}{4} H_{m}^{(2)}\left(k_{s} r_{s}\right) .\left(H_{m}^{(2)}(\bullet)\right.$ is the $m^{\text {th }}$ order Hankel function of the second kind).

The equation (3) have an infinite number of orthogonal modes, which is not possible to be achieved, therefore this series expansion can be truncated to a finite number due to the properties of the Bessel function within the region of interest which is a circular region of radius $r_{l}\left(\forall r \leq r_{l}\right)$. Hence, (3) can be truncated to $M$ terms as:

$$
P_{d}(\boldsymbol{x})_{K}=\sum_{s=1}^{S} \sum_{m=-M}^{M} \alpha_{m}^{s}\left(k_{s}\right) J_{m}\left(k_{s} r\right) e^{i m \phi}
$$

where the truncation order $M=\left\lceil e r_{r} k_{\max } / 2\right\rceil$ [13], $e=\exp (1)=2.718$ and $K_{\max }$ is the maximum wavenumber corresponding to maximum frequency. $J_{m}\left(k_{s} r\right) e^{i m \phi}$ is the basis function of cylindrical harmonic expansion.

By using (4), the far-field sound sources can be accurately reproduced. Nevertheless, this format does not work well for nearby sound field reproduction. To address this issue, a modified distance coding method is proposed in next subsection.

\subsection{Modified Distance Coding}

Virtual auditory environments (VAEs) based on HOA method can effectively reproduce the far-field sound sources. However, when the sound source is located within the loudspeaker array (i.e., it is nearby sound source), the quality of reproduced sound field between the interest region (the black circle in Figure1) and the loudspeaker array (the red circle in Figure1) degrades seriously. This phenomenon is called as near-field distortion as shown in Figure1. 
Considering some application areas (i.e., auditory display, vehicle simulators), it is important to eliminate the near-field distortion. In cylindrical harmonics analysis of HOA, virtual source encoding (i.e. positional encoding) includes distance coding and directional coding. From [6], it can be known that the near-field distortion is caused by the distance information; the reason is that the original virtual source encoding cause a finite, low frequency amplification which is negative for nearby sound sources.

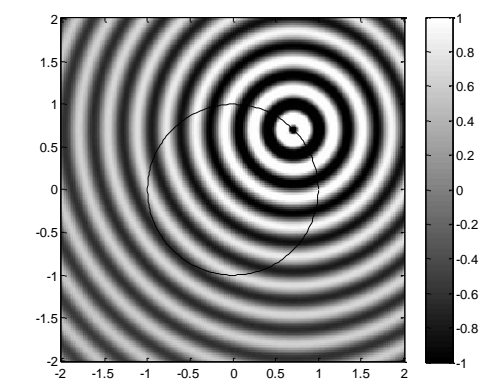

(a) Desired Sound Field

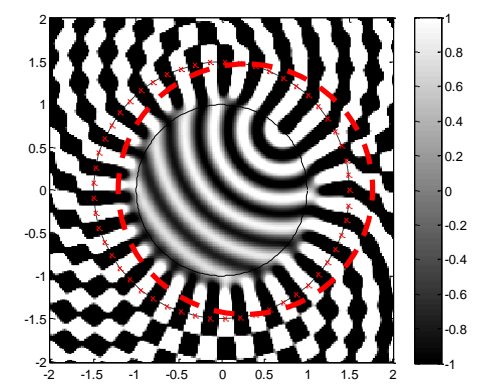

(b) Reproduced Sound Field

Figure 1. Near-field Distortion of HOA Method

To solve the above issues, an improved positional encoding equation is proposed in this paper, which is a modified form of the original encoding equation (4). The modified encoding coefficients are given as:

$$
\beta_{m}^{s}\left(k_{s}\right)=\psi_{s} \rho_{m}\left(k_{s}\right) E_{m}\left(\phi_{s}\right)
$$

where $s \in\{1,2, \ldots, S\}, m \in\{-M,-M+1, \ldots, M\}$. The modified factor $\rho_{m}\left(k_{s}\right)$ is described by

$$
\rho_{m}\left(k_{s}\right)=\frac{\mu_{2}}{\left|H_{m}^{N F C\left(r_{0}, R\right)}\left(k_{s}\right)\right|+\mu_{1}},
$$

where $\mu_{1}$ and ${ }^{\mu_{2}}$ are correction coefficients that need to be satisfied $\mu_{2}=1+\mu_{1}$. $H_{m}{ }^{N F C\left(r_{s}, r_{i}\right)}\left(k_{s}\right)$ which represents the near-field compensation (NFC) filter is expressed by

$$
H_{m}{ }^{N F C\left(r_{s}, r_{l}\right)}\left(k_{s}\right)=\frac{F_{m}\left(k_{s} r_{s}\right)}{F_{m}\left(k_{s} r_{l}\right)},
$$

where the transfer function $F_{m}\left(k_{s} r_{n}\right)$ (the subscript $n$ can be replaced by $s$ or $l$ ) is obtained as:

$$
F_{m}\left(k_{s} r_{n}\right)=\frac{H_{m}^{(2)}\left(k_{s} r_{n}\right)}{H_{0}^{(2)}\left(k_{s} r_{n}\right)}
$$

Based on the modified distance coding module, the flow process diagram of the positional encoding can be described by Figure2, which is the detailed explanation of the equation (6).

By applying the proposed encoding format (6) to the cylindrical harmonic expansion of the multi-frequency source (5), the expression of the compensated multi-frequency sound field is get as:

$$
P_{d}(\boldsymbol{x})_{K}=\sum_{s=1}^{S} \sum_{m=-M}^{M} \lambda_{s} \rho_{m}\left(k_{s}\right) \frac{i}{4} H_{m}^{(2)}\left(k_{s} r_{s}\right) e^{-i m \phi_{s}} J_{m}\left(k_{s} r\right) e^{i m \phi}
$$




\subsection{Calculating Loudspeakers Weights}

The key issue of the multi-frequency sound field reproduction is to design the weights of loudspeakers. Hence, this subsection will establish the corresponding relationship between desired sound field and the reproduced sound field.

The simple source approach of Kirchhoff-Helmholtz Integral shows that the sound field is generated by the boundary pressure in the area surrounded by loudspeakers [14].

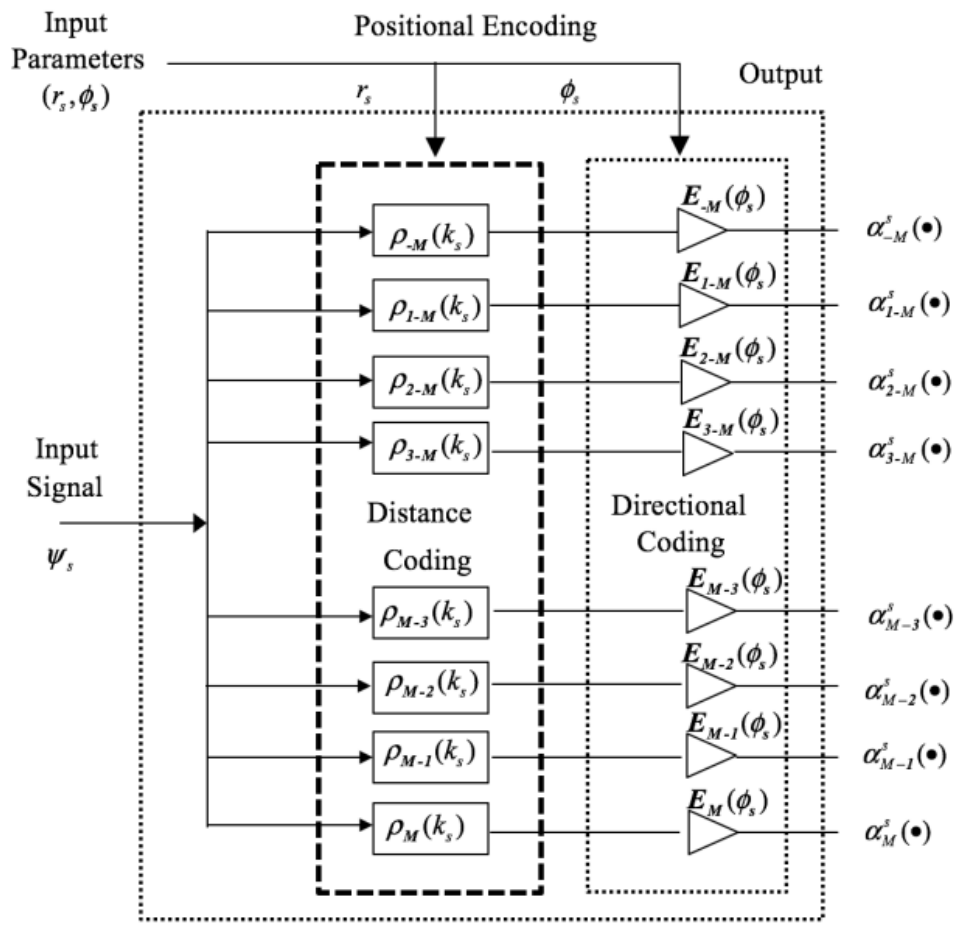

\section{Figure 2. NFC HOA Positional Encoding of a Virtual Sound Source: A Modified Distance Coding Unit Completes the Directional Encoding}

Therefore, given an observation point $\boldsymbol{x}=(r, \phi)$, a 2D loud-speaker positioned at $\boldsymbol{x}_{l}=\left(r_{l}\right.$, $\left.\phi_{l}\right)$ in the horizontal plane will radiate a sound field as:

$$
P_{l}\left(\boldsymbol{x}-\boldsymbol{x}_{l}, k\right)=\frac{i}{4} h_{0}^{(2)}\left(k\left|\boldsymbol{x}-\boldsymbol{x}_{l}\right|\right)
$$

where $k$ is the wavenumber satisfied $k=2 \pi f / c, f$ is the frequency.

Based on the addition theorem [11] for cylindrical harmonics, the 2D loudspeaker spatio-temporal transfer function (i.e., formula (11)) can be described by

$$
P_{l}\left(\boldsymbol{x}-\boldsymbol{x}_{l}, k\right)=\sum_{m=-M}^{M} \frac{i}{4} H_{m}^{(2)}\left(k r_{l}\right) e^{-i m \phi_{l}} J_{m}(k r) e^{i m \phi}
$$

Assume that $L$ number of such loudspeakers are employed in a circular discrete array where the $l^{\text {th }}(l=1,2, \ldots, L)$ loudspeaker is located at $\boldsymbol{x}_{l}=\left(r_{l}, \boldsymbol{\phi}_{l}\right)$. Therefore, the reproduced sound field can be represented as

$$
\begin{gathered}
P_{r}(\boldsymbol{x})_{K}=\sum_{s=1}^{S} \sum_{l=1}^{L} w_{l}\left(k_{s}\right) P_{l}\left(\boldsymbol{x}-\boldsymbol{x}_{l}, k_{s}\right) \\
=\sum_{s=1}^{S} \sum_{l=1}^{L} \sum_{m=-M}^{M} w_{l}\left(k_{s}\right) \frac{i}{4} H_{m}^{(2)}\left(k_{s} r_{l}\right) e^{-i m \phi_{l}} J_{m}\left(k_{s} r\right) e^{i m \phi}
\end{gathered}
$$

where $w_{l}\left(k_{s}\right)$ is the weight of the $l^{\text {th }}$ loudspeaker when the wavenumber is $k_{s}$. If $w_{l}\left(k_{s}\right)$ satisfy the condition that (13) equals to (10), an array of $L$ loudspeakers located on 
circular region of radius $r_{l}$, can exactly reproduce desired sound field at all observation points $r<r_{l}$, therefore, by minimizing the least squares errors between the desired sound field and the reproduction field sound field, i.e., $\left|P_{r}(\boldsymbol{x})_{K}-P_{d}(\boldsymbol{x})_{K}\right|^{2}$, is given by

$$
P_{r}(\boldsymbol{x})_{K}=P_{d}(\boldsymbol{x})_{K}
$$

By introducing (13) and (10) into (14), based the application of L'Hôpital's theorem, it can be obtained as

$$
\boldsymbol{H}_{s} \boldsymbol{w}_{s}=\boldsymbol{\beta}_{s}, s=1,2, \ldots, S
$$

where the weight vector $\boldsymbol{w}_{s}=\left\{w_{l}\left(k_{s}\right), w_{2}\left(k_{s}\right), \ldots, w_{L}\left(k_{s}\right)\right\}, \boldsymbol{\beta}_{s}=\left\{\beta_{-M}\left(k_{s}\right), \beta_{-M+1}\left(k_{s}\right), \ldots\right.$, $\left.\beta_{M}\left(k_{s}\right)\right\}$ is a vector representing the modified coefficients. The $s^{\text {th }}$ translation matrices $\boldsymbol{H}_{s}$ is attained as follow

$$
\boldsymbol{H}_{s}=\frac{i}{4}\left[\begin{array}{ccccc}
H_{-M}^{(2)}\left(k_{s} r_{l}\right) e^{i M \phi_{1}} & H_{-M}^{(2)}\left(k_{s} r_{l}\right) e^{i M \phi_{2}} & \cdots & \cdots & H_{-M}^{(2)}\left(k_{s} r_{l}\right) e^{i M \phi_{l}} \\
H_{-M+1}^{(2)}\left(k_{s} r_{l}\right) e^{i(M-1) \phi_{1}} & H_{-M+1}^{(2)}\left(k_{s} r_{l}\right) e^{i(M-1) \phi_{2}} & \cdots & \cdots & H_{-M+1}^{(2)}\left(k_{s} r_{l}\right) e^{i(M-1) \phi_{l}} \\
\vdots & \vdots & \vdots & \vdots & \vdots \\
H_{M}^{(2)}\left(k_{s} r_{l}\right) e^{-i M \phi_{1}} & H_{M}^{(2)}\left(k_{s} r_{l}\right) e^{-i M \phi_{2}} & \cdots & \cdots & H_{M}^{(2)}\left(k_{s} r_{l}\right) e^{-i M \phi_{l}}
\end{array}\right]
$$

According to $\boldsymbol{H}_{s}$ and $\boldsymbol{\beta}_{s}$, the required modal weights of loudspeakers can be obtained by

$$
\boldsymbol{w}_{s}=\boldsymbol{H}_{s}{ }^{\#}\left[\boldsymbol{H}_{s} \boldsymbol{H}_{s}{ }^{\#}+\gamma \boldsymbol{I}\right]^{-1} \boldsymbol{\beta}_{s}
$$

where \# represents the Hermitian Transpose, and $\boldsymbol{I}$ is a $[(2 M+1) \times(2 M+1)]$ identity matrix. Note that $\gamma$ is the regularization control parameter and $\gamma=0$ indicates the minimum energy solution of (17). To ensure the best reproduction performance, this paper sets $\gamma=0.001$.

\subsection{Influence of the Nearby Sound Source Position}

As shown in Subsection 2.2, it is worth noticing that the reproduction quality of the proposed positional encoding method is correlated with the position of the nearby sound source. Hence, to confirm the relationship between the reproduced error and the nearby sound position, a detailed analysis of the reproduced errors is given under the condition that the nearby sound sources are located at different positions.

The normalized mean square error (NMSE) is employed to describe the reproduced error in this paper, which is defined as follows:

$$
\varepsilon_{M}(\boldsymbol{x}, k)=\frac{\int_{0}^{r_{r}} \int_{0}^{2 \pi}\left|P_{d}(\boldsymbol{x}, k)-P_{r}(\boldsymbol{x}, k)\right|^{2} r d \phi d r}{\int_{0}^{r_{r}} \int_{0}^{2 \pi}\left|P_{d}(\boldsymbol{x}, k)\right|^{2} r d \phi d r}
$$

where $r_{r}$ is the radius of the reproduction region, $k=2 \pi f / c . P_{d}(\boldsymbol{x}, k)$ and $P_{r}(\boldsymbol{x}, k)$ represent the desired sound field and the reproduced sound field, respectively. $r_{r}$ is the radius of the reproduction region ( $r_{r}$ is set at $1 \mathrm{~m}$ in this paper), and $k$ is wavenumber. In this experiment, $L$ 2D loudspeakers are used, which are equally distributed on a circular array of radius $r_{l}=1.5 \mathrm{~m}$. Without loss of generality, a range of test frequencies (i.e., $600 \mathrm{~Hz}$, $1000 \mathrm{~Hz}, \ldots, 3800 \mathrm{~Hz}$ ) are chose in this experiment. Then, a general representation of the reproduction performance is given in Figure3 where NMSE is shown as a function of different nearby sound source distance which represents the position of the nearby sound source respect to the center of the region. From Figure3, it can be found that for different frequency sound sources, the reproduction errors of the proposed method decreases monotonically with the increase of the nearby sound source distance. Additionally, it is noted that when the nearby sound source position is equal to $1 \mathrm{~m}$ (i.e., the sound source is located at the boundary of the reproduced region), the reproduction error reaches its maximal value for most distances. Furthermore, the maximal reproduction error is 0.034 which agrees well with the expected value of 0.04 referring to [11], so the position of multi-frequency sound source is set at $1 \mathrm{~m}$ in following section. Nevertheless, it should be noted that the proposed method is not limited to reproducing such a position. For other positions, the reproduction errors only will be smaller than the selected position. 


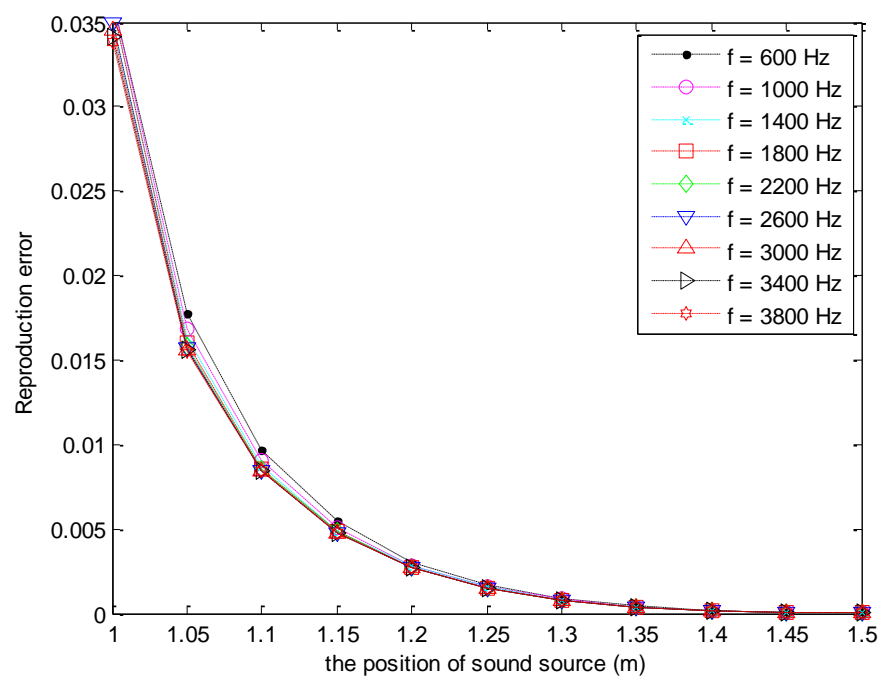

Figure 3. Reproduction Error of the Proposed Method at Different Positions of Nearby Sound Source

\section{Simulation Results}

In above section, the explicit interpretations of sound source position and the modified distance coding method are presented. In order to evaluate the reproduction performance of the proposed method, two simulation experiments are carried out and the corresponding results are given in this section. Firstly, the comparison experiment results are given over a referenced method. Secondly, the reproduction errors of sound field for various frequencies are evaluated. In these experiments, for convenience of the illustration purposes, the setting conditions are same to the above Subsection 2.4. The amplitudes (i.e., $\lambda_{1}, \lambda_{2}, \lambda_{3}, \ldots, \lambda_{s}$ ) are all set at 1 , but the proposed method is not limited to unit strength of each amplitude. The HOA method [11] with using original distance coding method for nearby sound source reproduction is employed as the referenced approach.

A) The first experiment: The simulation is used to demonstrate the effectiveness of the proposed method. For convenience of the illustration purposes, three-frequency sound source is located at $\left(1,3^{*} \pi / 2\right)$ is used, but the proposed method is not limited to threefrequency source. The frequency of the selected sound source consists of $500 \mathrm{~Hz}, 2000 \mathrm{~Hz}$ and $3500 \mathrm{~Hz}$. The number of loudspeakers $L$ is equal to 203 and hence the array is able to accurately produce a sound field with frequency up to $4000 \mathrm{~Hz}$ in a circular array of radius $r_{l}=1.5 \mathrm{~m}$.

The desired sound fields are shown in Figure 4. The loudspeaker weights are calculated from (17) and the reproduced sound field of referenced method and proposed method by the least squares method are shown in Figure 5 and Figure 6. Listening area is enclosed by a black circle in each. The loudspeakers are shown as " $x$ " marks. By comparing Figure 4 and Figure 6, it can be noted that the reproduced sound field of the proposed method corresponds well to the desired sound field. By comparing Figure 5 and Figure 6, it can be observed that the reproduced sound field within the interest region (the black circle) is almost identical with the referenced method. Nevertheless, the reproduced quality of sound field between the interest region and the loudspeaker array (the red circle) is significantly better than the referenced method. In addition, it can be observed that within outside loudspeaker array, the reproduced sound field is also obviously better than the referenced method. These simulation results validate the proposed method can effectively compensate the near-field distortion. 


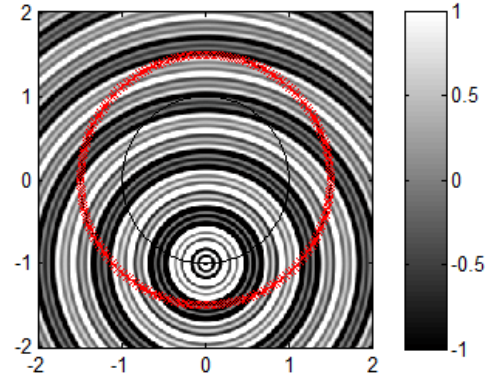

(a) Real

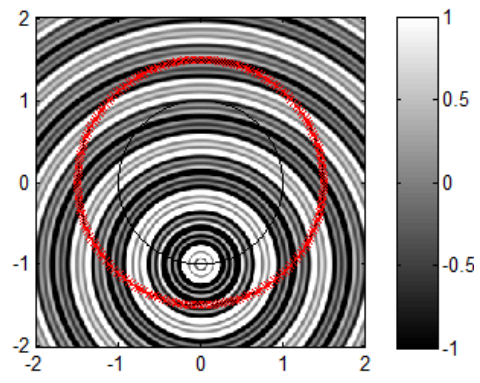

(b) Image

Figure 4. Desired Sound Field

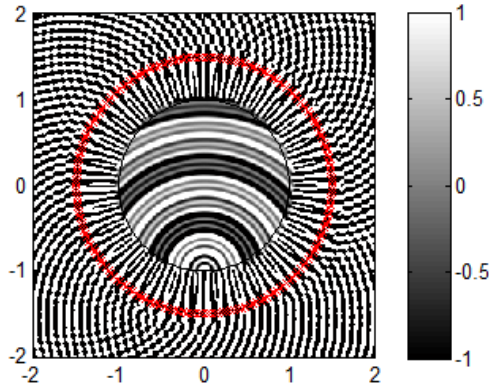

(a) Real

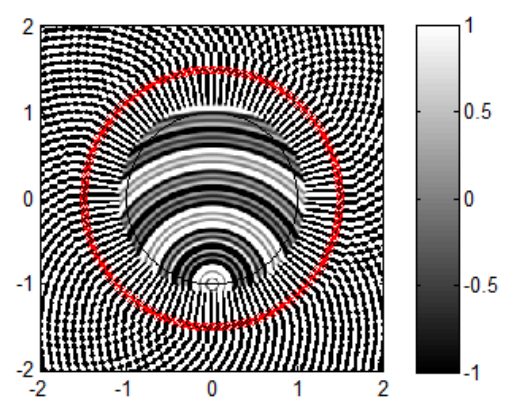

(b) Image

Figure 5. Reproduced Sound Field with Using Original Distance Coding Method

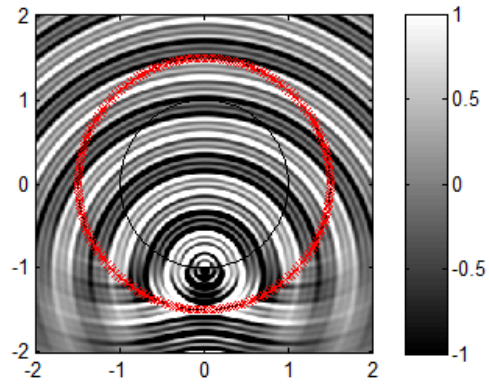

(a) Real

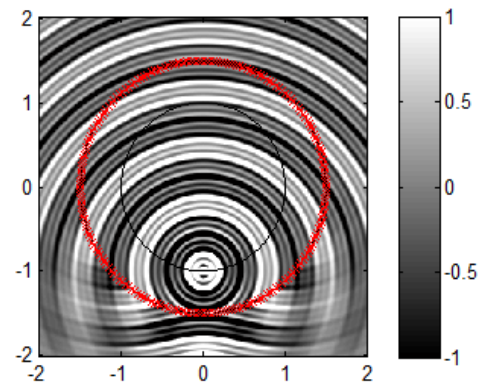

(b) Image

Figure 6. Reproduced Sound Field by Proposed Distance Coding Method

B) The second experiment: Additionally, in order to evaluate the reproduction performance of the proposed method for different frequency sound sources, the MSE (dB) is also computed. The MSE ( $\mathrm{dB})$ is defined as follows:

$$
\varepsilon_{M(\mathrm{~dB})}(\boldsymbol{x}, k)=10 \log _{10} \frac{\int_{0}^{r_{r}} \int_{0}^{2 \pi}\left|P_{d}(\boldsymbol{x}, k)-P_{r}(\boldsymbol{x}, k)\right|^{2} r d \phi d r}{\int_{0}^{r_{r}} \int_{0}^{2 \pi}\left|P_{d}(\boldsymbol{x}, k)\right|^{2} r d \phi d r}
$$

According to (18) and (19), two more general representations of the reproduction performance are given in Figure7 and Figure8. The Figure7 compares the reproduction errors of the proposed method and the referenced method within the interest region (i.e., the NMSE of two methods in the area $\left.\boldsymbol{D}_{\text {interest }}=\left\{\boldsymbol{x}(r, \phi) \mid r<r_{r}, l=1,2, \ldots, L\right\}\right)$. Furthermore, Figure8 describes the reproduction errors of two methods in exterior sound field (i.e., the NMSE (dB) of two methods in the area $\left.\boldsymbol{D}_{\text {exterior }}=\left\{\boldsymbol{x}(r, \phi) \mid r_{r}<r<r_{l}, l=1,2, \ldots, L\right\}\right)$. 


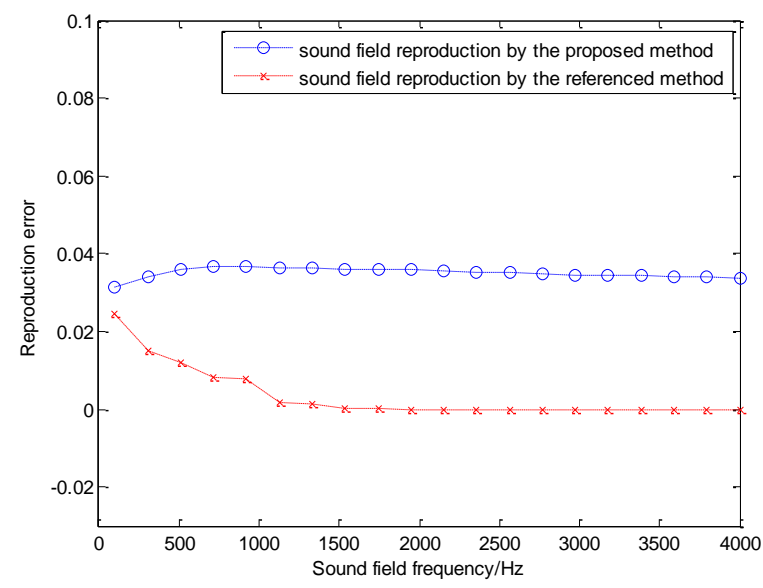

Figure 7. The Reproduction Error of Proposed Method and Referenced Method within the Interest Region

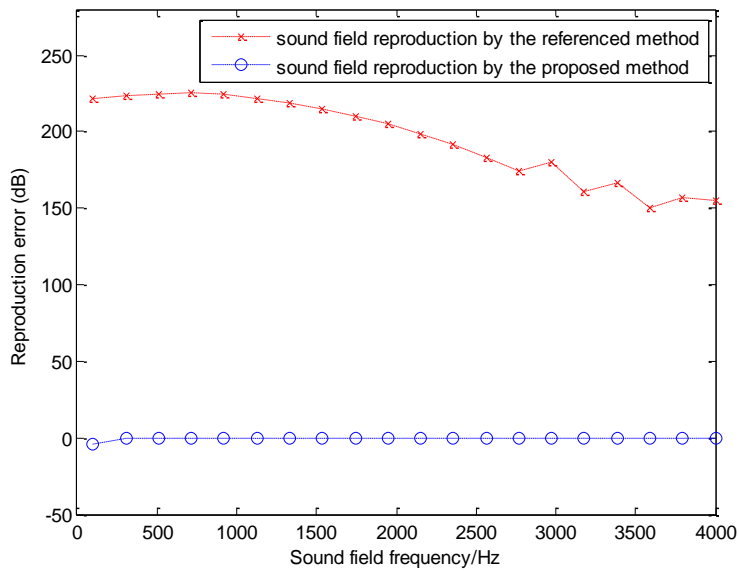

Figure 8. The Reproduction Error of Proposed Method and Referenced Method in Exterior Sound Field

In the Figure7, it can be seen that the reproduction errors of the proposed method are slightly larger than the referenced method in full frequency band. However, the reproduction errors of the proposed method are all less than 0.04 which is considered as error upper limit referring to [11]. Therefore, within the interest region, the nearby sound field is accurately reproduced by the proposed method.

From Figure8, it can be observed that the reproduction errors of the proposed method are significantly lower than the referenced approach in exterior sound field. The reason is that the proposed method eliminates near-field distortion and reproduces exterior sound field. In conclusion, it is worth recalling that the proposed method is more appropriate for nearby sound field reproduction. 


\section{Conclusion}

In this paper, based on the cylindrical harmonic synthesis of HOA, a new method for the nearby multi-frequency source reproduction is proposed. Firstly, to restrict the nearfield distortion and achieve an efficient implementation, a modified distance coding method is proposed. Secondly, by introducing the method, the modified cylindrical harmonic expansion of nearby multi-frequency source is obtained. Then, we use the least squares method to derive the loudspeaker driving signals. Simulation results show that the proposed method can accurately reproduce multi-frequency sound field and the near-field distortion is evidently eliminated; furthermore, the reproduction errors of exterior sound field are significantly lower than the referenced method. Therefore, the theory and the proposed modified distance coding method addressed in this paper can be useful in designing sound systems.

\section{Acknowledgments}

This work has been supported by the Project supported by Beijing Postdoctoral Research Foundation, National Natural Science Foundation of China (No 61231015, 61201197).

\section{References}

[1] D. Brungart and W. Rabinowitz, "Auditory localization of nearby sources: Head-related transfer functions", The Journal of the Acoustical Society of America, vol. 106, no. 3, (1999), pp. 1465-1479.

[2] M. A. Poletti, "Improved methods for generating focused sources using circular arrays", Proceedings of 133rd Conv. Audio Eng. Soc, San Francisco, CA, (2012).

[3] H. Wierstorf, A. Raake, M. Geier and S. Spors, "Perception of focused sources in Wave Field Synthesis", Journal of the Audio Engineering Society, vol. 61, no. 1/2, (2013), pp. 5-16.

[4] S. Spors and J. Ahrens, "Reproduction of Focused Sources by the Spectral Division Method", Proceedings of 4th International Symposium on Communications, Control and Signal Processing, Limassol, Cyprus, (2010).

[5] J.-W. Choi and Y.-H. Kim, "Sound Field Reproduction of a Virtual Source Inside a Loudspeaker Array with Minimal External Radiation”, IEEE trans. Audio, Speech, Lang. Process, vol. 21, no. 2, (2013), pp. 247-259.

[6] J. Daniel and S. Moreau, "Spatial sound encoding including near field effect: introducing distance coding filters and a viable, new Ambisonics format", Proceedings of AES 23rd Int. Conf.: Signal Process. Audio Recording Reproduction, Copenhagen, Denmark, (2003).

[7] J. Daniel and S. Moreau, "Further investigations of high order Ambisonics and wavefield synthesis for holophonic sound imaging", Proceedings of the 114th Audio Engineering Society Convention, (2003).

[8] J. Daniel, S. Moreau, "Further study of sound field coding with higher order Ambisonics", Proc. of the 116th Audio Engineering Society Convention, Berlin, Germany, (2004).

[9] J. Ahrens and S. Spors, "Spatial Encoding and Decoding of Focused Virtual Sound Sources", Proceedings of Ambisonics Symposium, Graz, Austria, (2009).

[10] S. Favrot and J. M. Buchholz, "Reproduction of nearby sound sources using higher-order Ambisonics with practical loud-speaker arrays", Acta Acustica united with Acustica, vol. 98, no. 1, (2012), pp. 48-60.

[11] Y. J. Wu and T. D. Abhayapala, "Theory and design of soundfield reproduction using continuous loudspeaker concept", IEEE Transactions on Speech and Audio Processing, vol. 17, no. 1, (2009), pp. 107-116.

[12] B. Bu, T. D. Abhayapala, C. C. Bao and W. Zhang, "Para-meterization of the three-dimensional room transfer function in horizontal plane", The Journal of the Acoustical Society of America, vol. 138, no. 3, (2015), pp. 280-286.

[13] M. S. Jia and W. B. Wang, "2.5D Sound Field Reproduction Using Higher Order Loudspeakers", Cybernetics and information technologies, vol.15, no. 6, (2015), pp. 5-15.

[14] J. Ahrens, "Analytic Methods of Sound Field Synthesis," Berlin, Germany: Springer Press, (2012). 


\section{Authors}
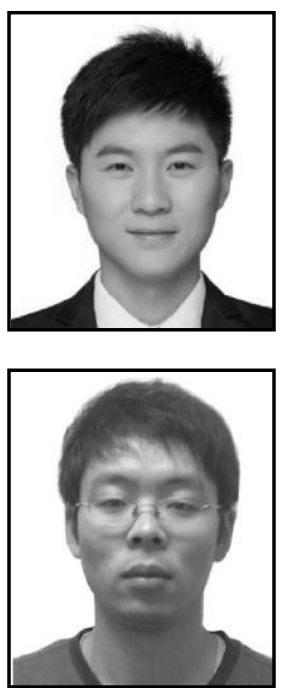

Maoshen Jia, received the B.E. degree in Electronic Information Engineering in 2005 from Hebei University, China. He received the Ph.D. degree in Electronic Science and Technology in 2010 from Beijing University of Technology, China. Recently, he is an associate professor of School of Electronic Information and Control Engineering in Beijing University of Technology. His current research interests include multichannel audio signal processing, sound field reproduction, source localization, source separation and scalable audio coding. He is a senior member of IEEE, a senior member of CIE, and a member of AES.

Jundai Sun, received the B.E. degree in Science and Technology of Electronic Information in 2015 from Agricultural University of Hebei, China. He is currently working towards the M.S. degree in Information and Communication Engineering at Beijing University of Technology, China. His current research interests include source localization and separation.

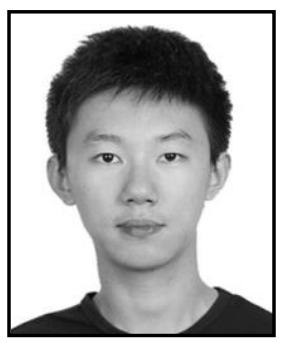

Jiaming Zhang, received the B.E. degree in Electronic Information Engineering in 2016 from Beijing University of Technology, China. He is currently working towards the M.S. degree in Information and Communication Engineering at Beijing University of Technology, China. His current research interests include source localization and separation. 
International Journal of Signal Processing, Image Processing and Pattern Recognition Vol. 10, No. 4 (2017) 